\title{
Spontaneous splenic rupture in an acute leukemia patient with splenic tuberculosis: A case report
}

\author{
YUE ZHANG $^{1}$, JUAN ZHANG ${ }^{1}$, TINGTING CHEN ${ }^{1}$, HUI ZENG ${ }^{1}$, BING ZHAO ${ }^{1}$, \\ YONG ZHANG $^{1}$, XIAOHUAN ZHOU ${ }^{1}$, WEI HAN ${ }^{2}$, YANPING HU ${ }^{3}$, FENGGE LIU ${ }^{3}$, \\ ZHIJUAN SHAN ${ }^{1}$, WEIFENG GAO ${ }^{1}$ and HEBING ZHOU ${ }^{1}$ \\ Departments of ${ }^{1}$ Hematology, ${ }^{2}$ General Surgery and ${ }^{3}$ Pathology, Luhe Hospital, \\ Capital Medical University, Beijing 101149, P.R. China
}

Received June 13, 2016; Accepted November 17, 2016

DOI: $10.3892 /$ mco. 2016.1120

\begin{abstract}
Spontaneous splenic rupture, also referred to as atraumatic splenic rupture, is a rare but life-threatening emergency condition. Without timely diagnosis and treatment, the mortality rate of splenic rupture approaches $100 \%$. The etiology of atraumatic splenic rupture varies; it is reportedly associated with neoplasms or splenic infection, but is rarely encountered in patients with both conditions. We herein report the case of a 58-year-old male patient with acute myeloid leukemia (AML) complicated by splenic tuberculosis (TB), who presented with spontaneous rupture of the spleen. Pathological examination of the resected spleen revealed multifocal granulomatosis with caseous necrosis. However, with timely diagnosis and surgical intervention, the patient recovered successfully and is currently on consolidation therapy. To the best of our knowledge, this is the first case of spontaneous splenic rupture in AML with splenic TB. The relevant literature on spontaneous splenic rupture was also reviewed and the potential etiology and treatment were discussed.
\end{abstract}

\section{Introduction}

Spontaneous splenic rupture, also referred to as atraumatic splenic rupture, is an uncommon serious complication of acute leukemia, with very few reported cases since Rokitansky first described spontaneous rupture of the spleen in a patient with leukemia in 1861 (1-7). Although the precise occurrence of spontaneous splenic rupture is not known, its actual morbidity may be higher due to the difficulty of diagnosis in the past. In the past, the mortality rate of splenic rupture was extremely high, due to the patient's poor general condition, complications

Correspondence to: Dr Hebing Zhou, Department of Hematology, Luhe Hospital, Capital Medical University, 82 Xinhua South Road, Tongzhou, Beijing 101149, P.R. China

E-mail: zhbyffs@126.com

Key words: spontaneous splenic rupture, acute leukemia, splenic tuberculosis and difficulty in diagnosis, with the major cause of death being hypotensive shock from the extensive blood loss, whereas infection and other complications also played a major role. However, with the increased availability of imaging modalities, the mortality rate has been reduced significantly over the past decades. In the present study, we report a case of spontaneous splenic rupture occurring in a patient with acute myeloid leukemia (AML).

\section{Case report}

A 58-year-old male patient was admitted to the Luhe Hospital (Beijing, China) in July, 2015, with a 2-month history of fever and cough, without symptoms of fatigue or night sweats. The patient had no history of TB or human immunodeficiency virus infection. The physical examination was unremarkable. The peripheral blood smear revealed pancytopenia [hemoglobin (HGB) level of $83 \mathrm{~g} / \mathrm{l}$, white blood cell (WBC) count of $2.92 \times 10^{9} / 1$ and platelet (PLT) count of $61 \times 10^{9} / 1$ ], with $22 \%$ blasts. Certain blast cells included Auer rods. The bone marrow smear was characterized by hypercellularity and patches of myeloblasts with Auer rods (Fig. 1). These results were consistent with AML of the M2 subtype. Following the diagnosis of leukemia, leukemia genetic screening was performed. The gene screening was negative. The c-KIT exon 8, c-KIT exon 17, FMS-like tyrosine kinase 3 internal tandem duplication and nucleophosmin 1 were all wild-type, but Janus kinase (JAK) 2V617F or calreticulin (CALR) gene detection was not performed. Bone marrow conventional cytogenetic analysis on the G-banded metaphases revealed the following: 46,XY, del (20)(q11)(20). The results of fluorescence in situ hybridization were as follows: AML1/ETO:RUNX/RUNX1T1 $0 \%$.

The patient achieved complete remission after induction chemotherapy with a regimen comprising daunorubicin $60 \mathrm{mg} / \mathrm{m}^{2}$ on days $1-3$ and cytarabine $200 \mathrm{mg} / \mathrm{m}^{2}$ on days 1-7; however, he developed severe myelosuppression, agranulocytosis, and was febrile for 3 weeks. The galactomannan assay was $56.10 \mathrm{pg} / \mathrm{ml}$. Bronchoalveolar lavage revealed a few bronchial epithelia and multifocal neutrophilic infiltration. An abdominal and pelvic CT scan revealed no hepatosplenomegaly. The patient was empirically treated with broad-spectrum antibiotics (imipenem and cilastatin sodium for injection, 


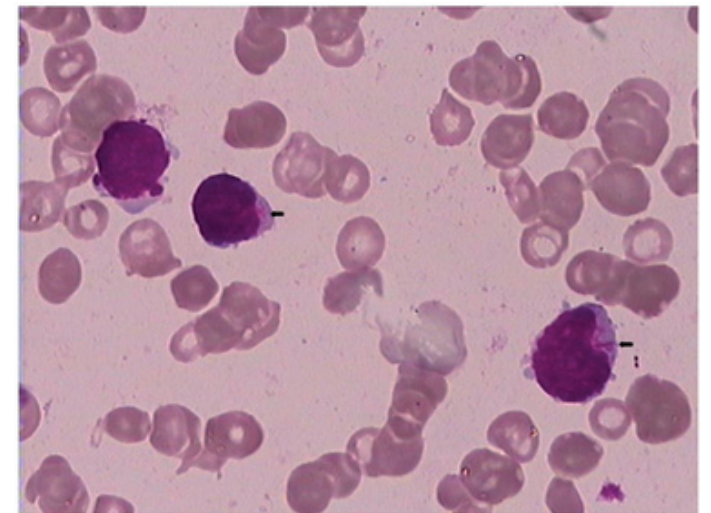

Figure 1. Bone marrow smear showing myeloblasts with Auer rods (arrows). Wright-Giemsa staining; magnification, x1,000.

vancomycin hydrochloride for injection, cefoperazone sodium and sulbactam sodium for injection) and antifungal agents [voriconazole for injection (DSM Pharmaceuticals, Inc., Greenville, NC, USA) and amphotericin B (North China Pharmaceutical Co., Ltd., Shijiazhuang, China)]. A repeat chest CT showed improvement of the infiltrates and his temperature normalized.
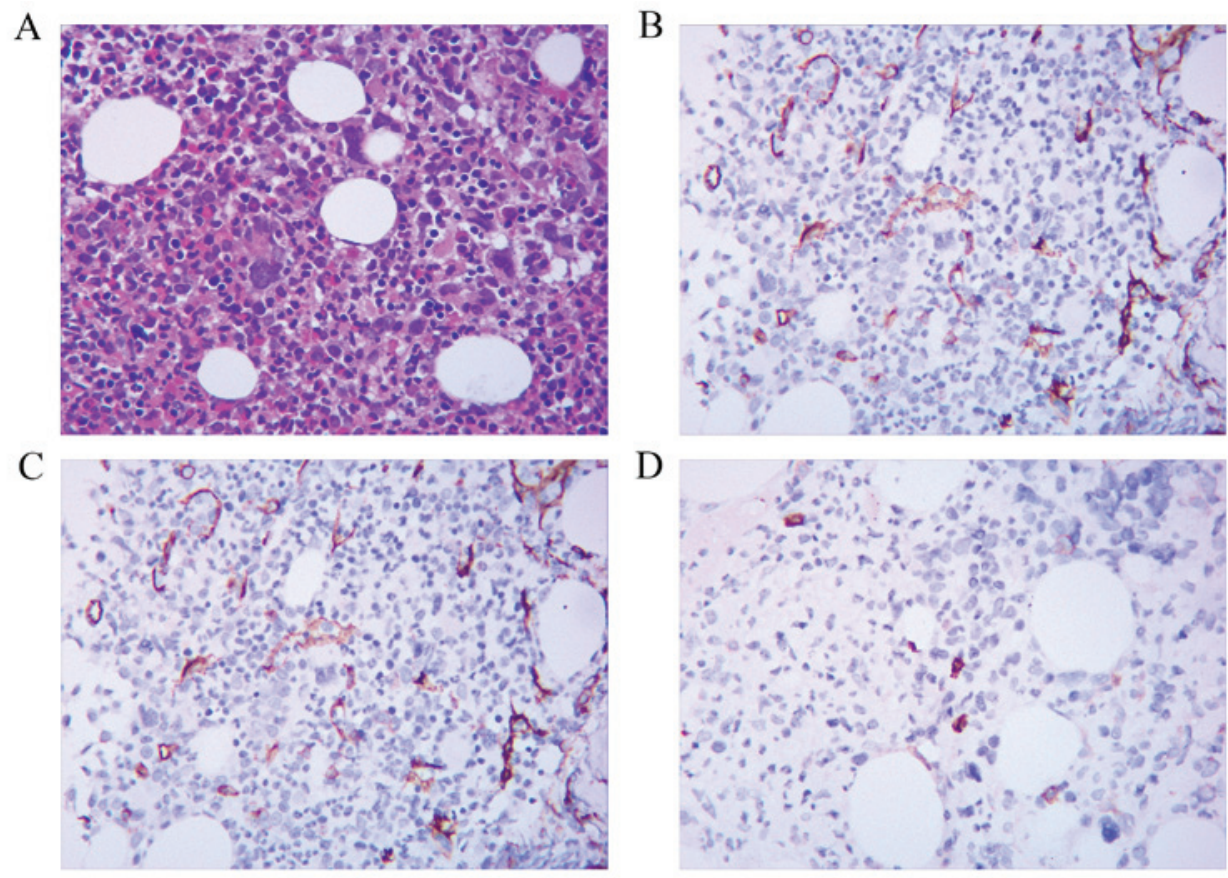

After the second course of chemotherapy, the peripheral blood smear revealed a WBC count of $4.84 \times 10^{9} / 1$ with a small number of immature WBCs, an HGB level of $106 \mathrm{~g} / \mathrm{l}$ and a PLT count of $335 \times 10^{9} / 1$. On repeat bone marrow biopsy, dry tap aspiration, focal granulopoietic progenitors and abnormal localization of immature precursors were observed. The red cell population consisted mainly of intermediate- and late-stage erythroblasts. The number of multinucleated megakaryocytes was increased (8-12/high-power field). The reticulin fibres were focally proliferated and were positive $(++)$ on Gömöri trichrome staining. On immunocytochemical staining, there was a small number of $\mathrm{CD} 34^{+}$precursor cells, the leukemic blasts were myeloperoxidase (MPO) ${ }^{+}$and fractional leukemic blasts were $\mathrm{CD} 117^{+}$. The Ki-67 index was 30\% (Fig. 2). Interestingly, a homozygous JAK2V617F mutation was detected by polymerase chain reaction (PCR), as well as a missense mutation in exon 9 of the CALR gene by bidirectional sequencing. The latter mutation has been classified as c.1142A $>\mathrm{C}$ (p.E381A). The results were consistent with AML with myelofibrosis (MF).

A third course of chemotherapy with mitoxantrone $4 \mathrm{mg} / \mathrm{m}^{2}$ on days $1-3$ and cytarabine $150 \mathrm{mg} / \mathrm{m}^{2}$ on days $1-7$ was subsequently administered. On day 3 , the patient developed sudden-onset nausea, dizziness and severe abdominal pain, he

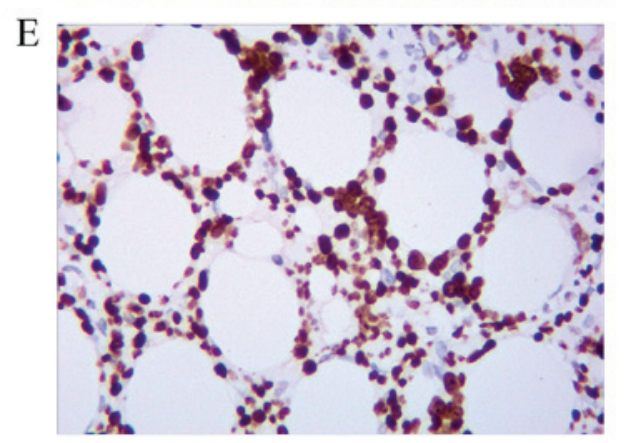

Figure 2. Histopathological bone marrow examination. (A) Hematoxylin and eosin staining (magnification, $\mathrm{x} 400$ ). Immunohistochemical staining for (B) CD34 (magnification, x400); (C) myeloperoxidase (magnification, x400); and (D) CD117 (magnification, x400). (E) Ki-67 (magnification, x400). 
A

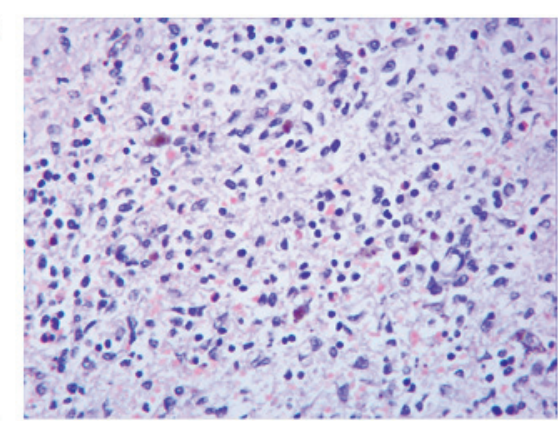

C

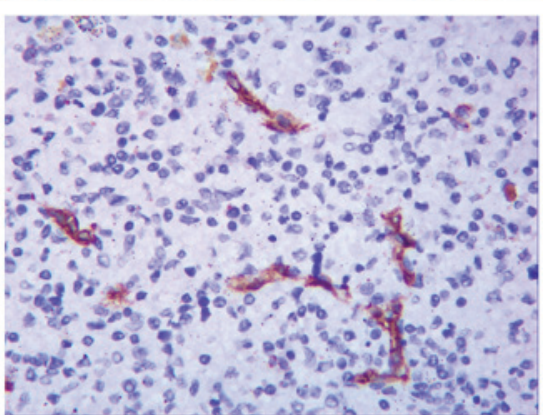

E

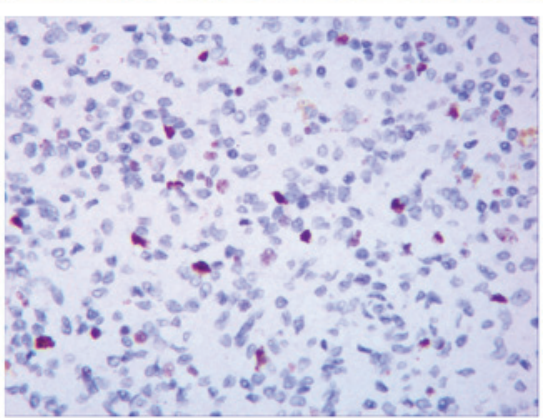

$\mathrm{B}$

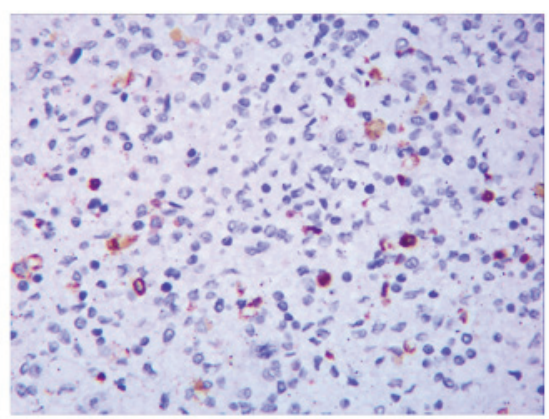

$\mathrm{D}$

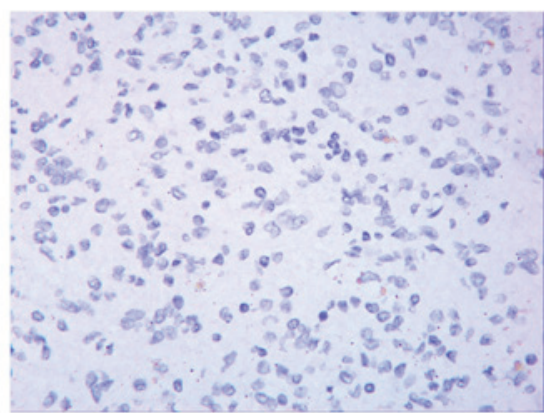

$\mathrm{F}$

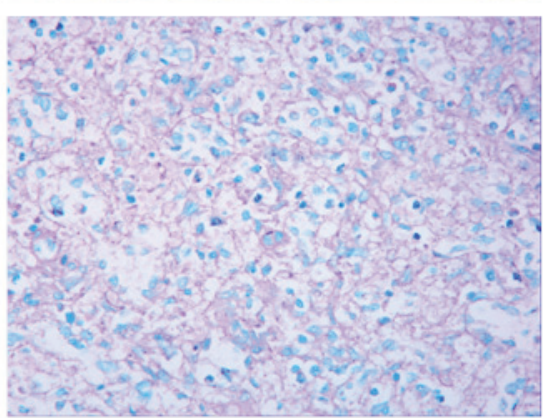

Figure 3. Histopathological examination of the splenic tissue. (A) Hematoxylin and eosin staining (magnification, $\mathrm{x} 400$ ). Immunohistochemical staining for (B) myeloperoxidase (magnification, x400); (C) CD34 (magnification, x400) and (D) CD117 (magnification, x400). (E) Ki-67 staining (magnification, x400. (F) Periodic acid Schiff staining (magnification, $\mathrm{x} 400$ ).

quickly became hypotensive and the HGB level decreased to $8.3 \mathrm{~g} / \mathrm{dl}$ within $1 \mathrm{~h}$. The coagulation tests (prothrombin time, activated partial thromboplastin time, thrombin time and fibrinogen) were within the normal range. The patient received aggressive fluid resuscitation, vasopressors (dopamine; Shanghai Hefeng Pharmaceutical Co., Ltd., Shanghai, China), and was intubated for respiratory support. Chemotherapy was discontinued after a total dose of $8 \mathrm{mg} / \mathrm{m}^{2}$ of mitoxantrone and $300 \mathrm{mg} / \mathrm{m}^{2}$ of cytarabine. An abdominal ultrasound revealed a considerable amount of free fluid surrounding the liver and spleen, with a density consistent with that of blood. An emergency laparotomy confirmed splenic rupture and splenectomy was performed during the procedure. Approximately 4,000 $\mathrm{ml}$ of fresh blood were evacuated from the abdominal cavity. The patient was intubated and transferred to the intensive care unit. After 1 week, the patient was extubated and he eventually recovered fully.

Pathological examination of the resected ruptured spleen revealed increased red pulp, decreased white pulp and multifocal granulomatosis with caseous necrosis, which raised the suspicion of TB; however, the acid-fast staining was negative. Furthermore, immunohistochemical staining of the splenic tissue revealed the presence of some MPO-positive cells, but they were negative for CD34 and CD117. The Ki-67 was sparsely positive in a few splenic cells. The periodic acid-Schiff staining was negative (Fig. 3). JAK2V617F mutation in the splenic specimens was negative by the PCR method.

A purified protein derivative test was strongly positive. The erythrocyte sedimentation rate was $128 \mathrm{~mm} / \mathrm{h}$. The T-SPOT. TB test was negative. The patient was empirically treated for TB with a combination of isoniazid, rifampicin, ethambutol and pyrazinamide, despite the acid-fast staining being negative. On bone marrow biopsy 1 month later, there was no dry tap aspiration and complete remission was achieved. However, JAK2V617 and CALR mutations were still detected. The patient then received consolidation chemotherapy with standard-dose cytarabine (Pfizer Italia Srl, Rome, Italy), daunorubicin (Haizheng Pharmaceutical Co., Ltd., Taizhou, China) or mitoxantrone (Jiangsu Hengrui Pharmaceutical Co., Lianyungang, China). The last follow-up was in October, 2016, and the patient exhibited complete remission of the bone marrow.

\section{Discussion}

Spontaneous splenic rupture refers to rupture of the spleen without a history of blunt or penetrating trauma. Although rare, spontaneous splenic rupture may be associated with 
neoplastic $(30.3 \%)$, infectious $(27.3 \%)$, inflammatory, non-infectious (20.0\%), drug- and treatment-related $(9.2 \%)$ and mechanical (6.8\%) disorders; it may also occur in the normal spleen (6.4\%) (8). The major symptoms and signs of splenic rupture include abdominal pain, tenderness and guarding, hypotension, nausea, vomiting, dizziness and syncope (9). However, no characteristic clinical manifestation may be used to definitively diagnose splenic rupture without further investigation. The definitive treatment for spontaneous splenic rupture is emergent splenectomy. Without splenectomy, the mortality rate in such patients approaches $100 \%$ (10).

While atraumatic splenic rupture most commonly occurs in association with neoplastic diseases, its mechanism has not been fully elucidated. In 1964, Hynes et al (11) proposed three possible mechanisms underlying spontaneous splenic rupture, which remain widely accepted to date: Mechanical effect of distention secondary to leukemic infiltration of the spleen, particularly the capsule; splenic infarct with capsular hemorrhage and subsequent rupture; and blood coagulation defects. However, it appears that none of the abovementioned mechanisms was responsible for the spontaneous splenic rupture in our patient. First, his spleen was not infiltrated by leukemic cells on pathological examination, which was confirmed by negative immunohistochemical staining for CD117 and $\mathrm{CD} 34$, and the splenic tissue was negative for the JAK2V617F mutation. Moreover, there was no evidence of blood coagulopathy or splenic infarct. Therefore, the patient's splenic rupture cannot be explained by leukemia or intracapsular hemorrhage.

The splenic rupture in our patient likely involved other underlying mechanisms. First, the pathological examination of the ruptured spleen revealed findings considered to be typical for TB. Splenic TB is an extremely rare cause of splenic rupture, with only 3 cases of splenic abscess and rupture as a consequence of TB infection described in the literature to date $(12,13)$. TB mostly locates primarily in the lungs, and splenic TB is a rare form of extrapulmonary localization. Immunocompromised patients, such as those with acquired immunodeficiency syndrome, have been reported to be at high risk for splenic TB (14), but the diagnosis may be challenging due to the non-specific clinical manifestations. The patient may suffer from fever, left upper quadrant abdominal pain, weight loss, diarrhea and, occasionally, ascites (15). However, in one-third of the cases, there were no abdominal symptoms, despite sonographic confirmation of a splenic lesion (16). Therefore, the diagnosis of splenic TB is difficult and is often missed or delayed. In patients with TB, splenic TB may present as multiple small hypoechoic lesions on abdominal sonography, or hypodense lesions on abdominal CT (17). Diagnosis is established by pathological examination of the fine-needle aspirate, splenic biopsy, or examination of the surgical specimen following splenectomy (18). However, invasive diagnostic procedures are difficult to perform in hematological patients due to the increased risk of bleeding and marked neutropenia following intensive chemotherapy. Therefore, management is largely dependent on clinical diagnosis when limited microbiological data are available (19).

On the other hand, the patient may be diagnosed with AML with primary MF (PMF). The bone marrow biopsy revealed dry tap aspiration, increased megakaryocyte number and focal hyperplasia of the fibrous tissue, and was positive for
Gömöri trichrome staining, JAK2V617 and CALR mutations. The activating mutation JAK2V617F is frequently found in myeloproliferative neoplasms (MPNs), including polycythemia vera, essential thrombocytosis and PMF (20). MPNs share an increased risk of thrombotic and hemorrhagic complications. Thrombosis occurs in less typical sites, including cerebral sinus vessels and splanchnic vessels (hepatic, portal, mesenteric and splenic veins). Emerging risk factors may include leukocytosis and presence of the JAK2V617F mutation, or an increase in its allelic burden. Hemorrhagic complications are likely associated with acquired platelet defects, such as acquired von Willebrand syndrome (21). Whether thrombotic and hemorrhagic complications caused by MPNs are associated with splenic rupture remains to be further elucidated. Although splenectomy is an effective treatment for MPN-related symptoms, it is also associated with significant operative morbidity, increased mortality, and even a potential increased risk of blast phase transformation in MF $(22,23)$. Most clinicians manage to avoid splenectomy in the majority of the patients.

In conclusion, it is hypothesized that the splenic rupture in our patient was caused by the subcapsular localization of tuberculous granulomatous tissue infiltrating the red pulp of the spleen, predisposing to tearing of the splenic capsule. The role of MPN in the splenic rupture may be another, although unlikely, possibility, since there was no evidence of coagulopathy. Regardless of the underlying mechanism, sudden onset of abdominal pain associated with symptomatic anemia or hypotension in a patient with leukemia warrants further investigation to exclude fatal splenic rupture.

\section{References}

1. Han JS, Oh SY, Kim SH, Kwon HC, Hong SH, Han JY, Park KJ and Kim HJ: A case of pathologic splenic rupture as the initial manifestation of acute myeloid leukemia M2. Yonsei Med J 51: 138-140, 2010.

2. Zimmer BM, Berdel WE, Ludwig WD, Notter M, Reufi B and Thiel E: Fatal spleen rupture during induction chemotherapy with rh GM-CSF priming for acute monocytic leukemia. Clinical case report and in vitro studies. Leuk Res 17: 277-283, 1993.

3. Kasper C, Jones L, Fujita Y, Morgenstern GR, Scarffe JH and Chang J: Splenic rupture in a patient with acute myeloid leukemia undergoing peripheral blood stem cell transplantation. Ann Hematol 78: 91-92, 1999.

4. Hernández R, del Cañizo MC, López C, González MI, Vázquez ML, Caballero MD and San Miguel JF: Pathologic rupture of the spleen during induction with ATRA in a patient with acute promyelocytic leukemia. Med Oncol 17: 337-339, 2000.

5. Tan A,Ziari M, Salman H, Ortega W and Cortese C: Spontaneous rupture of the spleen in the presentation of acute myeloid leukemia. J Clin Oncol 25: 5519-5520, 2007.

6. Singhal V, Kuiper J, Chavda K and Kashmer D: Spontaneous splenic rupture as first manifestation of acute myeloid leukemia: Case report and review of literature. J Clin Oncol 29: e576-e578, 2011.

7. Zeidan AM, Mitchell M, Khatri R, Itani D, Boikos S, Bose S, Lipsett P,Efron D, King KE, Gerber J and DeZern A: Spontaneous splenic rupture during induction chemotherapy for acute myeloid leukemia. Leuk Lymphoma 55: 209-212, 2014.

8. Renzulli P, Hostettler A, Schoepfer AM, Gloor B and Candinas D: Systematic review of atraumatic splenic rupture. Br J Surg 96: 1114-1121, 2009.

9. Giagounidis AA, Burk M, Meckenstock G, Koch AJ and Schneider W: Pathologic rupture of the spleen in hematologic malignancies: Two additional cases. Ann Hematol 73: 297-302, 1996.

10. Canady MR, Welling RE and Strobel SL: Splenic rupture in leukemia. J Surg Oncol 41: 194-197, 1989. 
11. Hynes HE, Silverstein MN and Fawcett KJ: Spontaneous rupture of the spleen in acute leukemia. Report of 2 cases. Cancer 17: 1356-1360, 1964.

12. Yeo HJ, Lee SY, Ahn E, Kim EJ, Rhu DG, Choi KU, Lee SE, Cho WH, Jeon D and Kim YS: Spontaneous splenic rupture as a paradoxical reaction during treatment for splenic tuberculosis. Tuberc Respir Dis (Seoul) 75: 218-221, 2013.

13. Safioleas MC, Stamatakos MC, Safioleas CM, Diab AI and Agapitos EB: Co-existence of spontaneous splenic rupture and tuberculosis of the spleen. Saudi Med J 27: 1588-1590, 2006.

14. Gotor MA, Mur M, Guerrero L, Aspiroz C, Romero D and Gimeno E: Tuberculous splenic abscess in an immunocompetent patient. Gastroenterol Hepatol 18: 15-17, 1995 (In Spanish).

15. Pramesh CS, Tamhankar AP, Rege SA and Shah SR: Splenic tuberculosis and HIV-1 infection. Lancet 359: 353, 2002.

16. Dixit R, Arya MK, Panjabi M, Gupta A and Paramez AR Clinical profile of patients having splenic involvement in tuberculosis. Indian J Tuberc 57: 25-30, 2010

17. Zhan F, Wang CJ, Lin JZ, Zhong PJ, Qiu WZ, Lin HH, Liu YH and Zhao ZJ: Isolated splenic tuberculosis: A case report. World J Gastrointest Pathophysiol 1: 109-111, 2010.

18. Nayyar V, Ramakrishna B, Mathew G, Williams RR and Khanduri P: Response to antituberculous chemotherapy after splenectomy. J Intern Med 233: 81-83, 1993.
19. Kwon JC, Kim SH, Park SH, Choi SM, Lee DG, Choi JH, Yoo JH, Kim YJ, Lee S, Kim HJ, et al: Clinical characteristics and the usefulness of the QuantiFERON-TB Gold In-Tube test in hematologic patients with hepatic or splenic lesions. Korean J Intern Med 28: 187-196, 2013.

20. Yoshiki Y, Asai T, Ichikawa M, Hangaishi A, Ota S, Imai Y, Takahashi T and Kurokawa M: A case of myeloid sarcoma with correlation to JAK2V617F mutation, complicated by myelofibrosis and secondary acute myeloid leukemia. Intern Med 50: 2649-2652, 2011

21. McMahon B and Stein BL: Thrombotic and bleeding complications in classical myeloproliferative neoplasms. Semin Thromb Hemost 39: 101-111, 2013.

22. Santos FP, Tam CS, Kantarjian H, Cortes J, Thomas D, Pollock R and Verstovsek S: Splenectomy in patients with myeloproliferative neoplasms: Efficacy, complications and impact on survival and transformation. Leuk Lymphoma 55: 121-127, 2014.

23. Melikian AL, Kolosova L, Sokolova MA, Kovrigina AM, Silaev MA, Giliazitdinova EA, Gemdzhian ÉG and Karagiulian SR: Role of splenectomy in the treatment of myelofibrosis. Ter Arkh 85: 69-76, 2013 (In Russian). 